\title{
La structure des solutions aqueuses
}

\author{
D.H. Powell
}

Department of Chemistry, University Science Laboratories, South Road, Durham DH1 3LE, U.K.

\begin{abstract}
Résumé : En commençant par l'étude par diffraction neutronique de la structure des liquides moléculaires puis de l'hydratation des ions en solution, ce cours montrera comment les principes présentés lors des cours précédents peuvent être appliqués à des systèmes aqueux. Des exemples tirés de la littérature seront utilisés pour illustrer les considérations expérimentales propre à ce domaine et le genre d'informations que nous pouvons obtenir. Ce cours montrera également l'applicaton de la diffraction neutronique à des systèmes d'intérêt biologique et environnemental et se terminera par un examen de la complémentarité fournie par la diffraction des rayons $\mathrm{X}$, l'EXAFS et la RMN.
\end{abstract}

\section{INTRODUCTION}

L'eau est assurément le milieu réactif le plus important pour la vie et pour l'interaction entre la vie et la géosphère. Il y a donc un besoin de connaître la structure de l'eau et, de plus, la structuration de l'eau autour d'entités dissoutes ou à des interfaces biologiques ou minérales. Je présenterai ici les principes qui ont permis à la diffraction neutronique de faire une contribution majeure dans ce domaine de la science. Je ne prétends pas faire une revue exhaustive, ou même objective, de ce sujet très large. Je me limiterai à montrer quelques exemples d'études en commençant par des études de la structure de l'eau pure, en passant par des solutions aqueuses de sels simples et en finissant par des ions en solution en présence de macromolécules biologiques ou de surfaces minérales. Je tenterai d'indiquer en même temps la complémentarité des informations obtenues par diffraction neutronique avec celles obtenues par d'autres méthodes expérimentales. Les exemples que je donnerai sont tous tirés d'études de l'eau où de solutions aqueuses, mais les méthodes présentées sont également applicables à d'autres liquides moléculaires et à des solutions non-aqueuses.

Nous avons vu dans les cours précédents que la structure d'un liquide composé de plusieurs types d'atomes peut être décrite par une série de fonctions de distribution de paires partielles, $g_{\alpha \beta}(r)$, qui représentent la probabilité relative de trouver un atome de type $\beta$ à distance radiale, $r$, d'un atome de type $\alpha$. Nous avons vu également que la diffraction neutronique nous permet d'obtenir un facteur de structure, $F(Q)$, et que par des méthodes de substitution isotopique nous pouvons en extraire des facteurs de structure partiels, $S_{\alpha \beta}(Q)$, qui sont les transformées de Fourier des $g_{\alpha \beta}(r)$. Pour des solutions d'un ou de plusieurs solutés dans un solvant, il y aura plusieurs fonctions de distributions de paires partielles qui définissent les corrélations solvant-solvant, soluté-solvant et soluté-soluté. Je vais considérer d'abord les corrélations solvant-solvant, qui définissent la structure moyenne du solvant, avant de passer aux corrélations soluté-solvant, qui décrivent la structuration du solvant autour du soluté, 


\begin{tabular}{cccccc}
\hline Isotope & Abondance $/ \%$ & $b_{\mathrm{c}} / \mathrm{fm}$ & $b_{\mathrm{I}} / \mathrm{fm}$ & $\sigma_{\mathrm{c}} /$ barn & $\sigma_{\mathrm{s}} /$ barn \\
\hline${ }^{\mathrm{T}} \mathrm{H}$ ou $\mathrm{H}$ & 99.985 & -3.74 & 25.3 & 1.76 & 82.0 \\
${ }^{2} \mathrm{H}$ ou D & 0.015 & 6.67 & 4.04 & 5.592 & 7.64 \\
${ }^{16} \mathrm{O}$ & 99.76 & 5.80 & 0 & 4.23 & 4.23 \\
${ }^{17} \mathrm{O}$ & 0.038 & 5.78 & 0.18 & 4.20 & 4.20 \\
\hline
\end{tabular}

Tableau 1 : Les abondances naturelles, longueurs de diffusion neutroniques et sections croisées cohérentes et incohérentes des plus importants isotopes de l'hydrogène et de l'oxygène. Informations prises de [1]

\section{LA STRUCTURE DU SOLVANT}

Pour des solutions aqueuses, nous avons trois fonctions de distribution de paires partielles solvantsolvant : $g_{\mathrm{OO}}(r), g_{\mathrm{OH}}(r)$ et $g_{\mathrm{HH}}(r)$. Nous pouvons écrire le facteur de structure d'une solution aqueuse comme :

$$
F(Q)=c_{\mathrm{O}}^{2} b_{\mathrm{O}}^{2}\left[S_{\mathrm{OO}}(Q)-1\right]+2 c_{\mathrm{O}} c_{\mathrm{H}} b_{\mathrm{O}} b_{\mathrm{H}}\left[S_{\mathrm{OH}}(Q)-1\right]+c_{\mathrm{H}}^{2} b_{\mathrm{H}}^{2}\left[S_{\mathrm{HH}}(Q)-1\right]+\ldots \ldots
$$

où les termes qu'il faudrait ajouter sont les termes soluté-solvant et soluté-soluté, qui seront relativement petits pour des solutions diluées. En regardant le tableau 1, nous pouvons constater que le proton $\left({ }^{1} \mathrm{H}\right.$ ou $\left.\mathrm{H}\right)$ et le deutéron $\left({ }^{2} \mathrm{H}\right.$ ou $\left.\mathrm{D}\right)$ ont des longueurs de diffusion cohérentes de signe opposé. En faisant des expériences utilisant comme solvants $\mathrm{H}_{2} \mathrm{O}, \mathrm{D}_{2} \mathrm{O}$ ou un mélange des deux, il serait donc possible théoriquement d'effectuer une séparation du moins partielle de ces trois $S_{\alpha \beta}(Q)$. De telles expériences de diffraction neutronique sont, malheureusement, rendues compliquées par quelques problèmes pratiques que je présenterai brièvement avant de montrer des exemples de résultats expérimentaux.

Le premier problème est que les fortes corrélations intramoléculaires (corrélations entre les positions des atomes au sein d'une molécule) donnent des oscillations de $F(Q)$ jusqu'à des valeurs de $Q$ élevées. Pour définir ces corrélations il serait donc nécessaire de mesurer $F(Q)$ à des valeurs élevées. Les atomes $\mathrm{O}$ et $\mathrm{H}$ dans une molécule d'eau vibrent autour de leurs distances interatomiques moyennes, $d_{\mathrm{OH}}$ et $d_{\mathrm{HH}}$. La contribution à $F_{a b}(Q)$ d'une distance intramoléculaire $d_{\alpha \beta}$ est souvent représentée par une fonction de la forme $[2,3,4]$

$$
\begin{aligned}
& S_{\alpha \beta}^{\text {intra }}(Q)-1=C_{\alpha \beta}\left(\frac{\sin Q d_{\alpha \beta}}{Q d_{\alpha \beta}}\right) \exp \left\{-\frac{1}{2} \gamma_{\alpha \beta}^{2} Q^{2}\right\} \\
& C_{\alpha \beta}=\frac{1}{c_{\mathrm{m}}} \times \frac{2}{n_{\alpha} n_{\beta}}
\end{aligned}
$$

où $c_{\mathrm{m}}$ est la fraction atomique d'un seul atome dans la molécule (dans le cas de l'eau $c_{\mathrm{m}}=c_{\mathrm{O}} \equiv c_{\mathrm{H}} / 2$ ) et $n_{\alpha}$ est le nombre d'atomes $\alpha$ dans la molécule. Dans le cas de l'eau, le facteur statistique $2 / n_{\alpha} n_{\beta}$ est égal à 1 pour $S_{\mathrm{OH}}^{\text {intra }}(Q)$ et $1 / 2$ pour $S_{\mathrm{HH}}^{\text {intra }}(Q)$. Le facteur $1 / c_{\mathrm{m}}$ n'apparait pas dans les références citées, puisque ses auteurs utilisent une définition de $S(Q)$ normalisé par molécule plutôt que par atome. La partie ( $\sin Q d_{\alpha \beta} / Q d_{\alpha \beta}$ ) est la contribution exacte pour une molécule totalement rigide, tandis que le 
facteur exponentiel modélise la vibration autour de la distance interatomique moyenne et s'appelle souvent un facteur Debye-Waller par analogie avec le cas cristallin ${ }^{1}$. La transformée de Fourier de $S_{\alpha \beta}^{\text {intra }}(Q)$, quand elle est présentée comme $\operatorname{rg}_{\alpha \beta}{ }^{\text {intra }}(r)$, est une Gaussienne à $r=d_{\alpha \beta}$ avec largeur à mihauteur $(2 \ln 2)^{1 / 2} \gamma_{\alpha \beta}$ [5]. Un pic relativement large en $g_{\alpha \beta}^{\text {intra }}(r)$ correspond donc à une décroissance exponentielle rapide des oscillations en $S_{\alpha \beta}{ }^{\text {intra }}(Q)$ lorsque $Q$ augmente. Contrairement, un pic fin en $g_{\alpha \beta}^{\text {intra }}(r)$ correspond à une faible décroissance des oscillations en $S_{\alpha \beta}{ }^{\text {intra }}(Q)$, les oscillations continueront alors à des valeurs de $Q$ élevées.

Dans le cas de l'eau ou d'une solution aqueuse, la contribution intramoléculaire à $F(Q)$ (équation 1) avec l'approximation de l'équation 2 sera

$$
F^{\text {intrat }}(Q)=2 c_{\mathrm{H}} b_{\mathrm{o}} b_{\mathrm{H}}\left(\frac{\sin Q d_{\mathrm{OH}}}{Q d_{\mathrm{OH}}}\right) \exp \left\{-\frac{1}{2} \gamma_{\mathrm{OH}}^{2} Q^{2}\right\}+c_{\mathrm{H}} b_{\mathrm{H}}^{2}\left(\frac{\sin Q d_{\mathrm{HH}}}{Q d_{\mathrm{HH}}}\right) \exp \left\{-\frac{1}{2} \gamma_{\mathrm{HH}}^{2} Q^{2}\right\}
$$

De telles expressions pour modéliser la contribution intramoléculaire à $F(Q)$ sont souvent nommées facteurs de forme moléculaire. Nous montrons dans la figure 1 les facteurs de forme moléculaire pour l'eau normale, $\mathrm{H}_{2} \mathrm{O}$, et l'eau lourde, $\mathrm{D}_{2} \mathrm{O}$, calculés en utilisant l'équation 3 avec des paramètres pour l'eau pure à $298 \mathrm{~K}$ pris de la littérature : $d_{\mathrm{OH}}=0.98 \AA, \gamma_{\mathrm{OH}}=0.07 \AA, d_{\mathrm{HH}}=1.56 \AA, \gamma_{\mathrm{HH}}=0.07 \AA$ [4]. ${ }^{2}$ Nous pouvons constater que les oscillations en $F^{\text {intra }}(Q)$ sont encore bien visibles à $Q=30 \AA^{-1}$. La troncation de $F(Q)$ pour un système aqueux à une valeur de $Q$ inférieure à $Q=30 \AA^{-1}$ introduirait donc des erreurs dans le $G(r)$ obtenu par transformation de Fourier. Un manque de définition de la partie intramoléculaire aura ainsi des conséquences sur notre connaissance de la structure intermoléculaire du solvant. Idéalement, nous devrions utiliser un diffractomètre capable de mesurer $F(Q)$ à des valeurs de $Q$ jusqu'à au moins $30 \AA^{-1}$, avec de plus un rapport signal-bruit suffisant pour distinguer les oscillations structurales. De telles conditions sont réalisées par exemple sur le diffractomètre SANDALS [6] installé à la source à spallation ISIS; la présence de neutrons très énergétiques dans le faisceau à impulsions permet d'obtenir des valeurs de $Q$ élevées. Les diffractomètres installés sur des sources à réacteur sont généralement plus limités en gamme đe $Q$. Dans ce cas, il est usuel d'ajuster un facteur de forme moléculaire à $F(Q)$ et de soustraire cette contribution avant la transformation de Fourier afin d'éviter des erreurs de troncation dans $G(r)$.

\footnotetext{
1 Notez qu'ici le facteur Debye-Waller représente la variation d'une distance interatomique, pondérée sur un ensemble, tandis que pour un cristal il représente la variation de la position d'un seul atome, pondérée sur le temps.

${ }^{2}$ Notez que le $F^{\text {intra }}(Q)$ pour le $\mathrm{H}_{2} \mathrm{O}$ est de phase opposée à celui du $\mathrm{D}_{2} \mathrm{O}$, ceci parce que la contribution de $d_{\mathrm{OH}}$ domine dans les deux cas et le facteur de pondération, $2 c_{\mathrm{H}} b_{\mathrm{O}} b_{\mathrm{H}}$, est négatif pour $\mathrm{le}_{2} \mathrm{O}\left(b_{\mathrm{H}}\right.$ étant négatif) et positif pour le $\mathrm{D}_{2} \mathrm{O}\left(b_{2} \mathrm{H}\right.$ étant positif).
} 


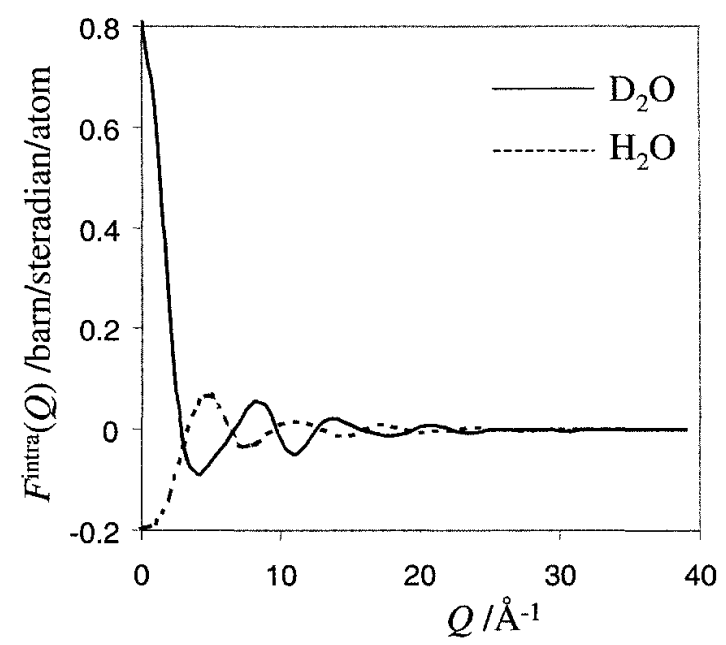

Figure 1 : Le facteur moléculaire de forme, $F^{\text {intra }}(Q)$, calculé pour l'eau normale et l'eau lourde.

Le deuxième problème expérimental que nous devons considérer si nous voulons faire des expériences de substitution isotopique pour l'hydrogène est la longueur de diffusion incohérente très élevée du proton. Nous avons vu que, dans l'approximation statique, l'intensité normalisée obtenue dans une expérience de diffraction neutronique peut être écrite comme suit :

$$
\begin{aligned}
& I(Q)=\frac{1}{N}\left(\frac{d \sigma}{d \Omega}\right)=\frac{1}{N}\left(\frac{d \sigma}{d \Omega}\right)^{\text {distincte }}+\frac{1}{N}\left(\frac{d \sigma}{d \Omega}\right)^{\text {self }} \\
& \frac{1}{N}\left(\frac{d \sigma}{d \Omega}\right)^{\text {distincte }}=F(Q)=\sum_{\alpha \beta} c_{\alpha} c_{\beta} b_{\alpha} b_{\beta}\left[S_{\alpha \beta}(Q)-1\right] \\
& \frac{1}{N}\left(\frac{d \sigma}{d \Omega}\right)^{\text {self }}=\sum_{\alpha} c_{\alpha} \overline{b_{\alpha}^{2}} \\
& b_{\alpha}=\left(b_{c}\right)_{\alpha} \\
& \overline{b_{\alpha}^{2}}=\left(b_{c}^{2}+b_{I}^{2}\right)_{\alpha}
\end{aligned}
$$

où $b_{\mathrm{c}}$ et $b_{I}$ sont les longueurs de diffusion respectivement cohérente et incohérente [1]. La partie distincte, autrement dit le signal de diffraction avec ses oscillations caractéristiques en $Q$, sera donc superposée à une contribution de diffusion self ${ }^{3}$, pouvant être considérée comme un signal de fond qui, dans l'approximation statique, ne varie pas avec $Q$. Dans le cas de l'eau isotopiquement 'normale',

\footnotetext{
${ }^{3}$ La diffusion dite 'self' (d'après le terme anglais 'self scattering') correspond à la diffusion par un ensemble d'atomes isolés, non corrélés dans l'éspace.
} 
${ }^{1} \mathrm{H}_{2} \mathrm{O}$ ou $\mathrm{H}_{2} \mathrm{O}$, la diffusion self sera particulièrement grande par rapport au signal de diffraction, à cause de la longueur de diffusion incohérente très élevée du proton. Ce signal de fond élevé a pour effet de compliquer légèrement l'expérience de diffraction (il faut utiliser des échantillons moins épais que d'ordinaire ${ }^{4}$ et compter plus longtemps pour avoir une qualité statistique comparable).

Ce problème de signal de fond se trouve malheureusement accru par notre troisième problème pratique, qui est que l'approximation statique des équations 4 n'est pas bonne pour des atomes légers comme l'hydrogène ou le deutérium. Plus l'atome est léger plus il y a un effet de recul de l'atome lors d'un événement de diffusion de neutron et donc plus l'échange d'énergie entre le neutron et l'atome est grand. Ce phénomène d'inélasticité a été décrit par Placzek [7] qui a proposé une méthode de correction adéquate pour des éléments relativement lourds. Cet 'effet de Placzek' est le plus marqué lorsque l'angle de diffusion est grand (recul de l'atome lors de la diffusion du neutron plus grand) et/ou l'énergie des neutrons incidents est faible (échange d'énergie entre le neutron et l'atome plus grand par rapport à l'énergie incidente). La manifestation de cet effet de Placzek sur l'intensité mesurée dépendra de la méthode utilisée pour mesurer la diffraction.

Dans le cas d'une source de neutrons continue (comme un réacteur) il est usuel d'utiliser un faisceau de neutrons incident monochromatique et un angle de diffraction variable. Dans ces conditions, l'effet de Placzek est plus grand à $Q$ élevé (plus grands angles de diffusion). Dans le cas d'une source à impulsions (comme la plupart des sources à spallation), il est en générale plus efficace de se servir de tout le faisceau polychromatique (ou 'blanc') et d'utiliser le temps d'arrivée des neutrons au détecteur pour définir leur énergie. Dans cette méthode dite 'temps de vol' il y a normalement des détecteurs à angle fixe et chaque détecteur couvre une gamme de $Q$ déterminée par l'énergie des neutrons incidents. La manifestation de l'effet de Placzek est alors plus compliquée que le cas monochromatique, puisque l'augmentation de $Q$ correspond en même temps à des angles plus élevés et des énergies plus élevées. Ceci est illustré par la Figure 2. Dans le cas des données obtenues du diffractomètre D20 de l'ILL (faisceau incident monochromatique et longueur d'onde de $0.95 \AA$ Å) les oscillations structurales en $r(Q)$ ne tournent pas autour d'un signal de fond plat, mais autour d'une fonction diminuant avec $Q$, suite à l'augmentation des effets de recul avec l'angle de diffusion. Dans le cas de l'eau normale, $\mathrm{H}_{2} \mathrm{O}$, le signal de fond de diffusion self est très grand, à cause de la grande section efficace incohérente du proton, et la pente de ce signal de fond très marquée à cause de la petite masse du proton. La très faible grandeur des oscillations par rapport à ce signal de fond démontre la difficulté à extraire l'information structurale pour des systèmes protonnés et explique pourquoi le $\mathrm{D}_{2} \mathrm{O}$ est souvent préféré comme solvant dans la diffraction neutronique. Le diffractomètre SANDALS à ISIS [6] a été conçu pour minimiser les effets d'inélasticité. Il n'utilise que des détecteurs à angles de diffusion relativement petits $\left(2 \theta<40^{\circ}\right)$ et exploite un faisceau à impulsions polychromatique

\footnotetext{
${ }^{4}$ Bien que la section efficace d'absorption du $\mathrm{H}_{2} \mathrm{O}$ ne soit pas grande, il y aura une forte atténuation du signal par un échantillon trop épais à cause de la diffusion multiple qui résultera de la section efficace de diffusion élevée.
} 
comprenant des neutrons de longueurs d'onde très petites (jusqu'à $0.05 \AA$ ) pour atteindre des valeurs de $Q$ relativement élevées. La figure $2 \mathrm{~b}$ montre que, même dans ces conditions, l'effet d'inélasticité n'est pas nul et que, à l'encontre du cas monochromatique, il est le plus grand à des valeurs de $Q$ petites.

Fort heureusement, l'effet de Placzek n'influence en première approximation que la diffusion self, l'effet sur la diffusion distincte étant négligeable [8]. ${ }^{5}$ Notre tâche est donc de soustraire de façon précise la contribution de la diffusion self, modifiée par l'effet d'inélasticité, afin d'obtenir $F(Q)$ et d'en extraire les informations structurales qui nous intéressent.

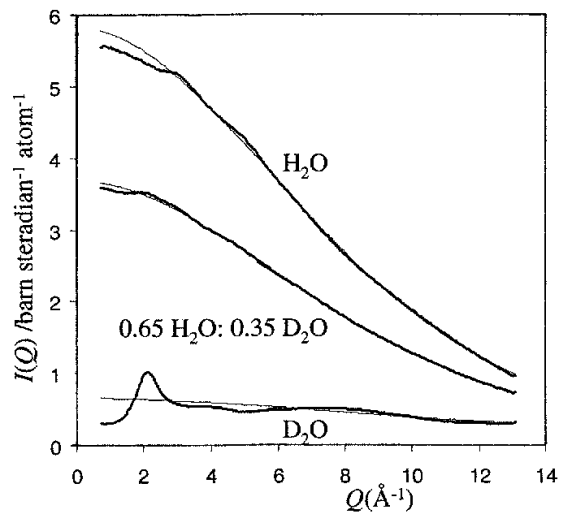

a)

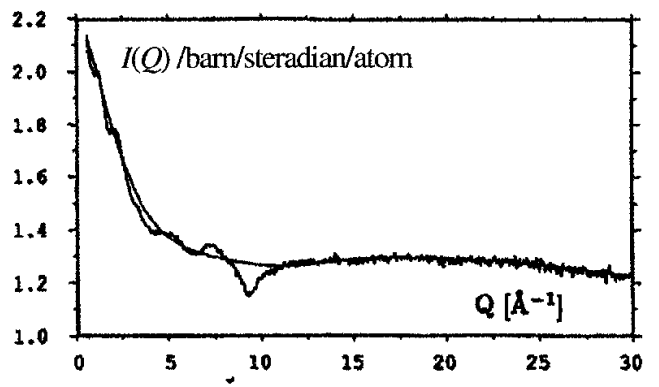

b)

Figure 2 : Intensité normalisée pour a) différentes compositions isotopiques de l'eau mesurée sur le diffractomètre D20 à l'ILL [9]et b) une solution de diméthyle sulfoxyde dans $l_{2} \mathrm{H}_{2} \mathrm{O}$ (dans un rapport $1: 1.85$ ) mesurée sur le diffractomètre SANDALS à ISIS [10]. Les courbes larges sont les données expérimentales et les courbes fines sont des estimations de la contribution de la diffusion self à $I(Q)$.

Pour l'instant, il n'y a pas de théorie qui permette de calculer les effets d'inélasticité sur la diffusion self dans des systèmes protonnés ou deutérés avec suffisamment de précision pour extraire $F(Q)$ de l'intensité mesurée, $I(Q)$. Il faudrait connaître le $S(Q, \omega)$ de l'échantillon (connaissance qui rendrait l'expérience superflue) ainsi que des paramètres expérimentaux tels que l'efficacité des détecteurs (en fonction de l'énergie) et le spectre en énergie du faisceau incident. Nous devons donc recourir à des méthodes semi-empiriques, où un polynôme (ou autre fonction comme la fonction

\footnotetext{
${ }^{5}$ Ceci peut s'expliquer de façon intuitive comme suit : la diffusion self correspond à la diffusion du neutron par un seul atome ; il y a donc un effet de recul de l'atome et un échange d'énergie appréciable. La diffusion distincte, par contre, correspond à une diffusion collective du neutron par tous les atomes dans la 'volume de cohérence' du neutron ; le transfert de moment lors de la diffusion est donc partagé par cet ensemble d'atomes et l'effet de recul de l'ensemble ainsi que le transfert d'énergie du neutron à l'ensemble sont par conséquent beaucoup plus faibles.
} 
pseudovoigt [11]) est ajusté à $I(Q)$ de façon à suivre la variation lente en $Q$ de la diffusion self, sans suivre la variation relativement rapide en $Q$ de $F(Q)$. On peut ajouter des critères pour mieux contraindre cet ajustement, comme par exemple le fait que $g_{\alpha \beta}(r)$ doive être égal à zéro en dessous d'une distance minimale d'approche. Les détails des différentes méthodes utilisées dépassent le cadre de ce cours, mais sont bien décrites dans la littérature [3,9].

Un développement récent intéressant est l'utilisation de méthodes dites 'inverses' qui permettent à la fois de déterminer $g_{\alpha \beta}(r)$ et de modéliser la structure du liquide. Les méthodes RMC (Reverse Monte Carlo) [12] et EPSR (Empirical Potential Structure Refinement) [13] utilisent des méthodes Monte Carlo pour simuler la structure du liquide en utilisant l'accord avec les données de diffraction comme contrainte. Dans la première méthode, qui sera présentée en détail dans un cours ultérieur, l'énergie d'interaction est remplacée par la déviation entre les facteurs de structure calculés à partir de la structure simulée et les facteurs de structure mesurés. Dans la deuxième méthode, une perturbation paramétrique est ajoutée à un potentiel de référence afin d'optimiser l'accord entre la structure simulée et les facteurs de structure mesurés. Soper [13] a utilisé la EPSR pour obtenir les fonctions de distribution de paires partielles pour l'eau pure à partir de données obtenues dans des expériences à faisceau monochromatiques (d'une source à réacteur) et polychromatiques (d'une source à impulsions). L'accord excellent entre les fonctions obtenues à partir des deux séries de données différentes (figure 3a) montre qu'il est possible d'obtenir des résultats structuraux très fiables, malgré les complications expérimentales décrites ci-dessus.

La figure $3 \mathrm{~b}$ montre la relation entre la structure locale de l'eau et les pics qui apparaissent dans les $g_{\alpha \beta}(r)$ de figure 3a. Les pics très fins à env. $1.0 \AA$ et $1.5 \AA$ respectivement dans $g_{\mathrm{OH}}(r)$ et $g_{\mathrm{HH}}(r)$ correspondent aux distances intramoléculaires. Le pic à $1.85 \AA$ en $g_{\mathrm{OH}}(r)$ est la corrélation intermoléculaire la plus forte et correspond au pont hydrogène entre un atome d'hydrogène d'une molécule d'eau et un atome d'oxygène d'une molécule adjacente. Les pics en $g_{00}(r)$ correspondent aux distances moyennes entre une molécule centrale et les molécules d'eau dans les première, deuxième et troisième sphères de coordination. La figure $3 \mathrm{~b}$ montre comment les pics dans les $g_{\alpha \beta}(r)$ définissent l'orientation relative des molécules voisines. La simulation EPSR produit des configurations en trois dimensions, et permet donc de visualiser de façon plus détaillée des configurations moléculaires physiquement raisonnables qui sont en accord avec les données expérimentales. La figure $3 \mathrm{c}$ montre clairement la structure quasi-tétraédrique de la première sphère de coordination et la tendance des molécules de la deuxième sphère d'hydratation à remplir les espaces laissés par la première sphère. Le pic à $4.5 \AA$ Aans $g_{00}(r)$, qui correspond à la distance de la deuxième sphère de coordination, peut être considéré comme la signature de la coordination tétraédrique entre les molécules d'eau.

La figure 4 a montre l'effet de l'introduction d'un soluté sur la fonction $g_{00}(r)$ de l'eau. L'effet le plus marqué est la disparition progressive du pic à $r=4.5 \AA$ et l'apparition d'un pic à $r \sim 3.4 \AA$ lorsque la concentration du soluté augmente. Un effet très semblable est obtenu quand l'eau pure est mise sous pression (figure 4b). La structure quasi-tétraédrique de l'eau, par analogie avec la glace, est une 
structure très ouverte. La haute pression a tendance à forcer l'eau à adopter une structure plus compacte, donc à détruire la structure tétraédrique. Il semble que le soluté, en construisant des molécules d'eau dans des sphères d'hydratation, empêche l'eau d'adopter la configuration quasitétraédrique qui est favorisée dans le liquide pur.

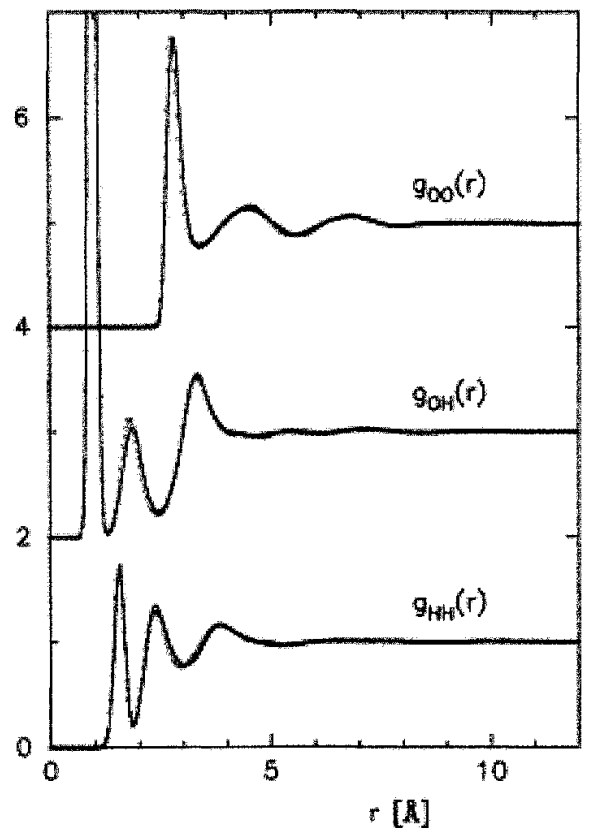

a)

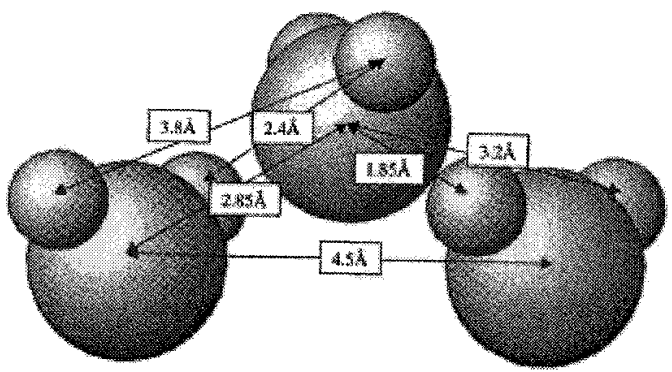

b)

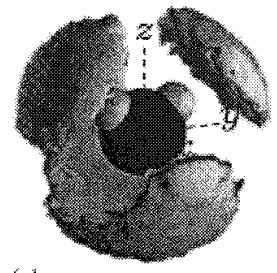

(a)

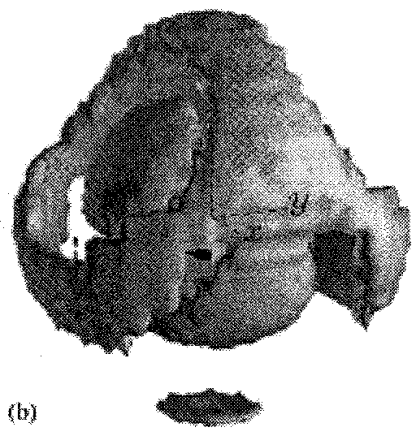

c)

Fig. 3 : (a) Fonctions de distribution de paires partielles de l'eau pure obtenues par la méthode EPSR à partir de mesures de diffraction neutronique avec faisceau incident monochromatique avec $\lambda=0.966 \AA$ (points) et mesuré en temps-de-vol (courbes) [13] (b) Représentation de la relation entre la structure locale de l'eau et les pics dans les $g_{\alpha \beta}(r)$ [14] (c) Graphiques de contours de la fonction de densité spatiale de l'eau autour de l'eau dans l'eau pure montrant la première et la deuxième sphère de coordination [14]. 


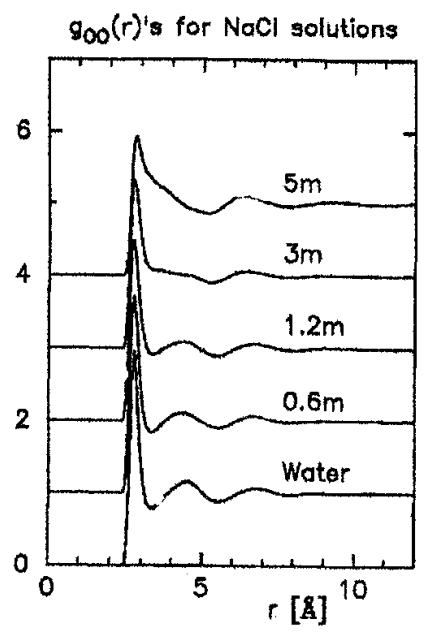

a)

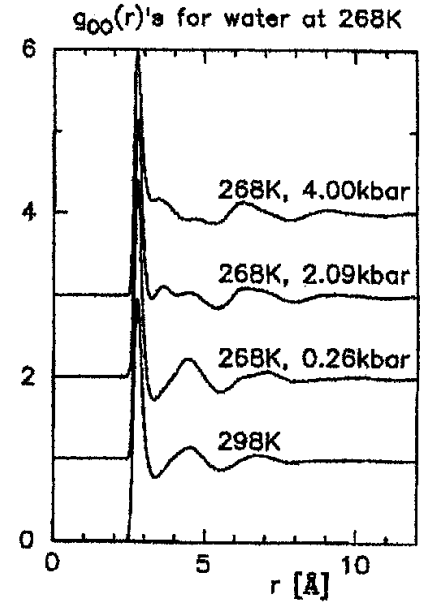

b)

Figure 4 : Comparaison entre l'effet de la concentration de $\mathrm{NaCl}$ (a) et de la pression (b) sur la fonction de distribution de paires partielle, $g_{0 o}(r)$ de l'eau [14]

\section{LA STRUCTURE SOLUTE-SOLVANT}

Considérons le cas d'un soluté $\mathrm{X}$ dans l'eau. Il y aura deux fonctions de distribution de paires partielles décrivant la structure du solvant autour de $\mathrm{X}: g_{\mathrm{XO}}(r)$ et $g_{\mathrm{XH}}(r)$. Nous aimerions alors pouvoir extraire les facteurs de structure partiels, $S_{\mathrm{XO}}(Q)$ et $S_{\mathrm{XH}}(Q)$ d'une expérience de diffraction. Si l'élément $\mathrm{X}$ possède deux isotopes ayant des longueurs de diffusion neutronique suffisamment distinctes nous pouvons déjà faire une séparation partielle en faisant deux expériences avec des solutions d'une même composition chimique et de compositions isotopiques de $\mathrm{X}$ différentes. Si la longueur de diffusion de $\mathrm{X}$ est de $b_{\mathrm{X}}$ dans la première solution et $b_{\mathrm{X}}$ ' dans la deuxième, d'après l'équation 4 la différence, $\Delta \mathrm{X}(Q)$ entre les deux intensités normalisées est :

$\Delta_{\mathrm{X}}(Q)=c_{\mathrm{X}}\left(\overline{b_{\mathrm{X}}^{2}}-\overline{b_{\mathrm{X}}^{2}}\right)+c_{\mathrm{X}}^{2}\left(b_{\mathrm{X}}^{2}-b_{\mathrm{X}}^{2}\right)\left(s_{\mathrm{Xx}}(Q)-1\right]+\sum_{\alpha \neq \mathrm{X}} 2 c_{\mathrm{x}} c_{\alpha}\left(b_{\mathrm{x}}-b_{\mathrm{x}}, b_{\alpha}\left[s_{\mathrm{X} \alpha}(Q)-1\right]\right.$

où $\alpha$ représente ici tous les types d'atomes dans le système sauf $X$. Le premier terme de cette équation représente la contribution de la diffusion self. Notons que la grande partie de la diffusion self (et surtout la partie problématique due a l'hydrogène) disparaît dans la différence : il ne nous reste que la différence de la diffusion self des deux isotopes de X. De plus, il a été démontré [15] que nous pouvons négliger l'effet d'inélasticité sur ce terme restant : nous retrouvons donc un signal de fond plat que nous pouvons aisément soustraire de $\Delta_{\mathrm{X}}(Q)$. Les deux autres termes dans l'équation 5 sont une somme 
pondérée des facteurs de structure partiels soluté-solvant et soluté-soluté. La transformée de Fourier de $\Delta_{\mathrm{X}}(Q)$, que nous écrirons $G_{\mathrm{X}}(r)$

$$
G_{\mathrm{X}}(r)=c_{\mathrm{X}}^{2}\left(b_{\mathrm{X}}^{2}-b_{\mathrm{X}}^{2}\right)\left[g_{\mathrm{XX}}(r)-1\right]+\sum_{\alpha \neq \mathrm{X}} 2 c_{\mathrm{X}} c_{\alpha}\left(b_{\mathrm{X}}-b_{\mathrm{X}}\right) b_{\alpha}\left[g_{\mathrm{X} \alpha}(r)-1\right]
$$

sera donc une somme pondérée de fonctions de distribution de paires partielles centrées sur le soluté $\mathrm{X}$. Nous arrivons ainsi à faire une séparation des $S_{\alpha \beta}(Q)$ ou $g_{\alpha \beta}(r)$ suffisante pour se concentrer sur la structure autour du soluté tout en éliminant les contributions gênantes de la diffusion self. Cette méthode s'appelle la méthode de différences de premier ordre, et les fonctions $\Delta_{\mathrm{X}}(Q)$ et $G_{\mathrm{X}}(r)$ sont des fonctions de différence de premier ordre. Dans bien des cas, cette méthode est adéquate pour définir la structure du solvant autour du soluté. Nous pouvons aussi procéder à une différence de deuxième ordre qui permet de séparer par exemple $S_{\mathrm{XH}}(Q)$ où $S_{\mathrm{XX}}(Q)$, mais de telles mesures sont très exigeantes au niveau expérimental et se limitent encore à quelques études.

\begin{tabular}{cccccc}
\hline Isotope & Abondance $/ \%$ & $b_{\mathrm{c}} / \mathrm{fm}$ & $b_{\mathrm{l}} / \mathrm{fm}$ & $\sigma_{\mathrm{s}} /$ barn & $\sigma_{\mathrm{a}} /$ barn $(\mathrm{a} 1.8 \AA)$ \\
\hline${ }^{\text {nat }} \mathrm{Ni}$ & & 10.3 & & 18.5 & 4.49 \\
${ }^{58} \mathrm{Ni}$ & 68.27 & 14.4 & 0 & 26.1 & 4.6 \\
${ }^{62} \mathrm{Ni}$ & 3.59 & -8.7 & 0 & 9.5 & 14.5 \\
${ }^{35} \mathrm{Cl}$ & 75.77 & 11.65 & 6.1 & 21.8 & 44.1 \\
${ }^{37} \mathrm{Cl}$ & 24.23 & 3.08 & 0.1 & 1.19 & 0.433 \\
${ }^{\text {nat }} \mathrm{Ar}$ & & 1.909 & & 0.683 & 0.675 \\
${ }^{36} \mathrm{Ar}$ & 0.337 & 24.9 & 0 & 77.9 & 5.2 \\
${ }^{n a t} \mathrm{~K}$ & & 3.67 & & 1.96 & 2.1 \\
${ }^{41} \mathrm{~K}$ & 6.73 & 2.69 & 1.5 & 1.2 & 1.46 \\
\hline
\end{tabular}

Tableau 2 : Les abondances naturelles, longueurs de diffusion neutroniques cohérentes et incohérentes et sections efficaces de diffusion et d'absorption des plus importants isotopes de quelques éléments considérés en tant que solutés dans ce cours. Informations prises de [1].

Considérons le cas des solutions aqueuses du chlorure de nickel. Le tableau 2 montre que le nickel et le chlore ont tous les deux des isotopes avec une différence de longueurs de diffusion neutronique appréciable (c'est pourquoi ce système a été le sujet de nombreuses études). La figure 5 montre les intensités normalisées mesurées pour des solutions de $1 \mathrm{~mol} \mathrm{~kg}^{-1}$ de $\mathrm{NiCl}_{2}$ dans le $\mathrm{D}_{2} \mathrm{O}$ avec deux compositions isotopiques différentes de nickel [9]. Nous voyons que, même dans ce système relativement concentré ayant un bon contraste entre les deux isotopes, la différence entre les deux intensités n'est pas très grande. Il est donc nécessaire pour de telles mesures de faire une préparation très soigneuse des échantillons, pour que la fonction de différence isotopique ne soit pas masquée par des différences chimiques entre les échantillons. Tout particulièrement, étant donnée la section efficace de diffusion très élevée du proton, il faut minimiser la contamination des échantillons deutérés par le 
$\mathrm{H}_{2} \mathrm{O}$ et surtout s'assurer que le contenu en $\mathrm{H}_{2} \mathrm{O}$ des deux échantillons est identique. Dans le cas présenté, la contamination en $\mathrm{H}_{2} \mathrm{O}$ (mesurée par absorption en infrarouge) était inférieure à $0.4 \%$ et la différence en contenu de $\mathrm{H} 2 \mathrm{O}$ entre les deux échantillons était inférieure à $0.2 \%$. Nous voyons aussi sur la figure 5 que l'intensité oscille autour d'un niveau qui, à petit $Q$, est proche du niveau de la diffusion self calculé dans l'approximation statique, mais que ce niveau diminue fortement à $Q$ élevé. Par contre, la fonction de différence de premier ordre (bas de la figure 5) oscille autour d'un niveau plat qui est proche du niveau de la diffusion self résiduel calculé selon l'équation 5 . Nous pouvons donc éviter une correction de la dépendance en $Q$ de la diffusion self ; la petite différence entre le niveau de $\Delta_{\mathrm{Ni}}(Q)$ et le niveau calculé est facilement corrigée en ajoutant une constante à $\Delta_{\mathrm{Ni}}(Q)$.

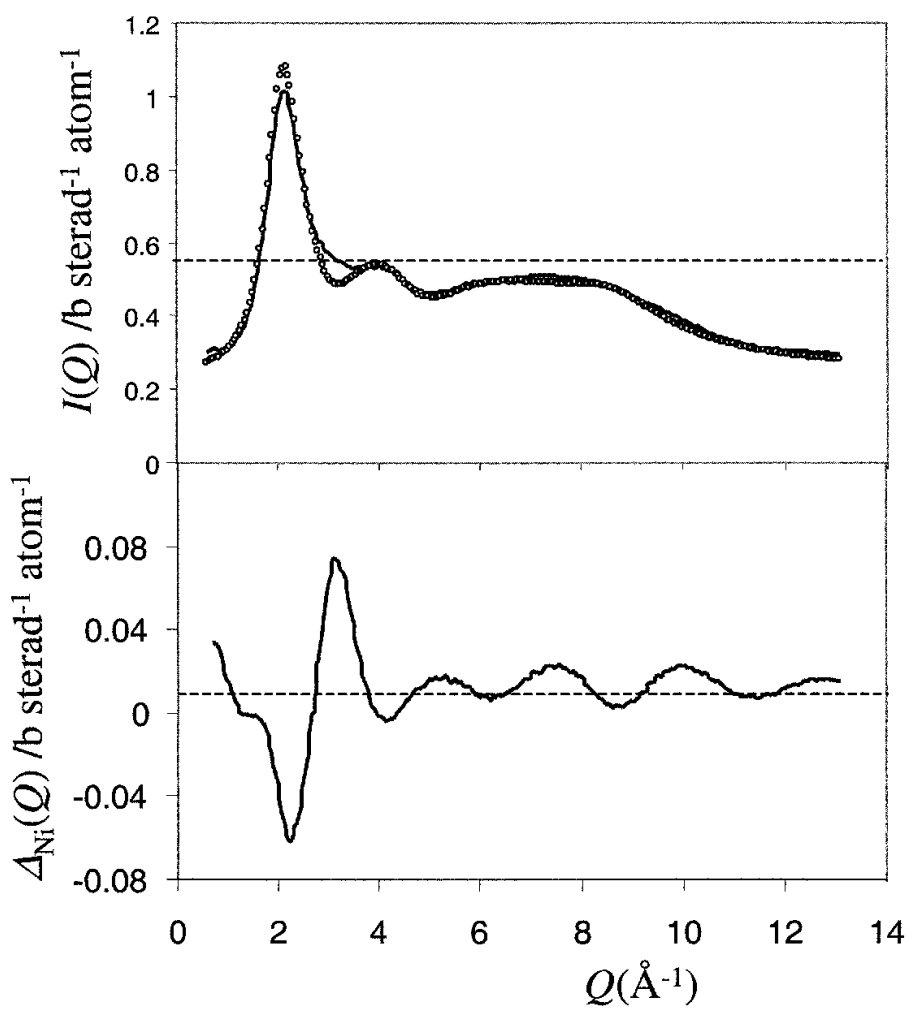

Figure 5 : En haut, les intensités normalisées mesurées sur le diffractomètre D20 de l'LLL avec une longueur d'onde incidente de $0.94 \AA$ pour des solutions $2.0 \mathrm{~mol} \mathrm{~kg}$ en ${ }^{\text {nat }} \mathrm{NiCl}_{2}$ (courbe) et ${ }^{62} \mathrm{NiCl}_{2}$ (points) dans le $\mathrm{D}_{2} \mathrm{O}$. En bas, la fonction de différence de premier ordre, $\Delta_{\mathrm{Ni}}(Q)$.[9] Les droites en pointillés représentent la contribution de la diffusion self aux intensités totales et à la différence calculée dans l'approximation statique. 


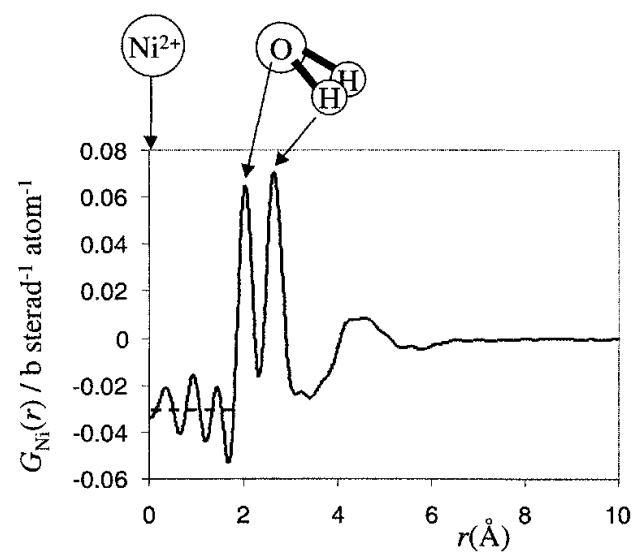

a)

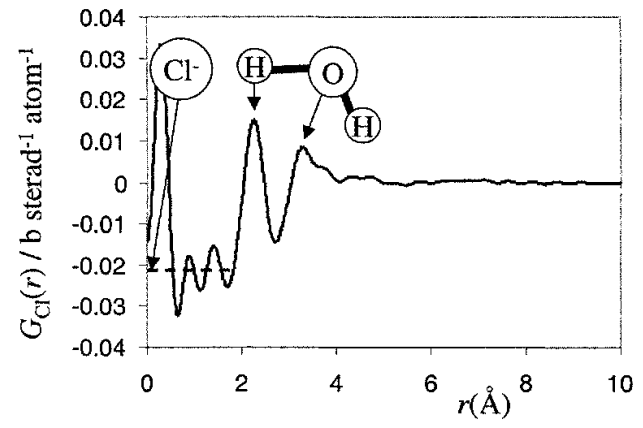

b)

Figure 6: Fonctions de différence de premier ordre, $G_{\mathrm{Ni}}(r)$ et $G_{\mathrm{C}}(r)$, pour des solutions $2.0 \mathrm{~mol} \mathrm{~kg}^{-1}$ en $\mathrm{NiCl}_{2}$ dans le $\mathrm{D}_{2} \mathrm{O}$.[9] Les droites en pointillés représentent la valeur de $G(r)$ calculé en dessous d'une distance d'approche physiquement raisonnable. Les dessins montrent comment les pics dans $G_{\mathrm{Ni}}(r)$ et $G_{\mathrm{Cl}}(r)$ correspondent à des distances ion-O et ion-H dans la première sphère d'hydratation des deux ions.

La transformée de Fourier, $G_{\mathrm{Ni}}(r)$, et la fonction analogue obtenue par substitution isotopique du chlore, $G_{\mathrm{Cl}}(r)$, sont montrées dans la figure 6. D'après l'équation 6 nous obtenons :

$G_{\mathrm{Ni}}(r)=8.499\left[g_{\mathrm{NiO}}(r)-1\right]+19.431\left[g_{\mathrm{NiH}}(r)-1\right]+1.121\left[g_{\mathrm{NiCl}}(r)-1\right]+0.081\left[g_{\mathrm{NiNi}}(r)-1\right]$ $G_{\mathrm{Cl}}(r)=6.238\left[g_{\mathrm{ClO}}(r)-1\right]+14.264\left[g_{\mathrm{CHH}}(r)-1\right]+0.399\left[g_{\mathrm{CNi}}(r)-1\right]+0.570\left[g_{\mathrm{CICl}}(r)-1\right]$

où les facteurs de pondération sont en unités de millibarn steradian ${ }^{-1}$ atom $^{-1}$. Les facteurs de pondération des termes soluté-soluté $\left(g_{\mathrm{NiCl}}(r)=g_{\mathrm{CINi}}(r), g_{\mathrm{NiNi}}(r)\right.$ et $\left.g_{\mathrm{ClCl}}(r)\right)$ étant relativement faibles, $G_{\mathrm{Ni}}(r)$ et $G_{\mathrm{Cl}}(r)$ nous informent sur la structure de l'eau autour des deux ions. ${ }^{6}[16]$ Les modèles de la figure 6 montrent comment les pics observés dans des fonctions de différence de premier ordre correspondent à des configurations de la première sphère d'hydratation raisonnable où une molécule d'eau pointe un oxygène vers le cation et un hydrogène vers l'anion. Les positions des pics nous donnent des distances moyennes ion-oxygène et ion-hydrogène. Si les pics sont bien résolus, comme c'est le cas pour les deux premiers pics de $G_{\mathrm{Ni}}(r)$ et le premier de $G_{\mathrm{Cl}}(r)$, nous pouvons aussi obtenir des nombres de coordination en utilisant

$$
n_{\alpha}^{\beta}=4 \pi \rho_{\mathrm{n}} c_{\beta} \int_{0}^{r_{\min }} g_{\alpha \beta}(r) r^{2} d r
$$

\footnotetext{
${ }^{6}$ Il faut ajouter que, dans le cas où il y aurait une forte tendance de l'anion à se lier au cation, les termes soluté-soluté ne peuvent pas être négligés, même si leurs facteurs de pondération sont faibles. Ceci à été observé dans le cas de solutions aqueuses du chlorure de zinc [16].
} 
où $n_{\alpha}^{\beta}$ est le nombre moyen d'atomes de $\beta$ à des distances radiales inférieure à $r_{\min }$ autour d'un atome central de type $\alpha, r_{\text {min }}$ est la distance au minimum après le premier pic en $g_{\alpha \beta}(r)$ et $\rho_{\mathrm{n}}$ est la densité d'atomes. Puisque $G_{X}(r)$ est une fonction composée nous devons combiner l'équation 7 avec l'équation 6 pour obtenir

$$
\begin{aligned}
& n_{\mathrm{Ni}}^{\mathrm{O}}=\frac{2 \pi \rho_{\mathrm{n}}}{c_{\mathrm{Ni}} b_{\mathrm{Ni}} b_{\mathrm{O}}} \int_{r_{1}}^{r_{2}}\left[G_{\mathrm{Ni}}(r)-G_{\mathrm{Ni}}(0)\right] r^{2} d r \\
& n_{\mathrm{Ni}}^{\mathrm{H}}=\frac{2 \pi \rho_{\mathrm{n}}}{c_{\mathrm{Ni}} b_{\mathrm{Ni}} b_{\mathrm{H}}} \int_{r_{1}}^{r_{1}}\left[G_{\mathrm{Ni}}(r)-G_{\mathrm{Ni}}(0)\right] r^{2} d r \\
& n_{\mathrm{Cl}}^{\mathrm{H}}=\frac{2 \pi \rho_{\mathrm{n}}}{c_{\mathrm{Cl}} b_{\mathrm{Cl}} b_{\mathrm{H}}} \int_{r_{\mathrm{i}}}^{r_{2}}\left[G_{\mathrm{Cl}}(r)-G_{\mathrm{Cl}}(0)\right] r^{2} d r
\end{aligned}
$$

où $G_{X}(0)$ est obtenu en substituant $g_{X a c}(0)=0$ dans l'équation 6

$$
G_{\mathrm{X}}(0)=-c_{\mathrm{X}}^{2}\left(b_{\mathrm{X}}^{2}-b_{\mathrm{X}}^{2}\right)-\sum_{\alpha \neq \mathrm{X}} 2 c_{\mathrm{X}} c_{\alpha}\left(b_{\mathrm{X}}-b_{\mathrm{X}}\right) b_{\alpha}
$$

et les valeurs de $r_{1}$ et $r_{2}$ dans les équations (8) délimitent le pic correspondant dans $G_{\mathrm{X}}(r)$. Les paramètres structuraux obtenus pour la solution aqueuse de $\mathrm{NiCl}_{2}$ sont donnés dans le tableau 3 . Les résultats indiquent donc que le cation et l'anion sont hydratés tous les deux par six molécules d'eau dans la première sphère d'hydratation. Pour le $\mathrm{Ni}^{2+}$, le fait que $n_{\mathrm{Ni}}{ }^{\mathrm{H}}=2 \times n_{\mathrm{Ni}}{ }^{\mathrm{O}}$ (comme attendu, puisqu'une molécule d'eau consiste en un oxygène et deux hydrogènes) prouve que les deux premiers pics de $G_{\mathrm{Ni}}(r)$ correspondent bien aux corrélations $\mathrm{Ni}-\mathrm{O}$ et $\mathrm{Ni}-\mathrm{H}$ comme indiqué sur la figure 6 . Dans le cas de $\mathrm{Cl}^{-}$la preuve de notre modèle sur la base de la différence de premier ordre est moins évidente, mais ce modèle a été prouvé par des expériences de différence de deuxième ordre où la substitution isotopique sur le chlore a été combinée avec la substitution isotopique $\mathrm{H} / \mathrm{D}$ pour obtenir $g_{\mathrm{ClH}}(r)$.[17]

\begin{tabular}{ccc}
\hline $\mathrm{X} \alpha$ & $r_{\mathrm{X} \alpha}(\AA)$ & $n_{\mathrm{X}}{ }^{\alpha}$ \\
\hline $\mathrm{NiO}$ & 2.06 & 5.9 \\
$\mathrm{NiH}$ & 2.67 & 11.7 \\
$\mathrm{ClH}$ & 2.28 & 5.7 \\
\hline
\end{tabular}

Tableau 3: Les paramètres structuraux (distances moyennes et nombres de coordination) des premières sphères d'hydratation du $\mathrm{Ni}^{2+}$ et du $\mathrm{Cl}^{-}$obtenus par des différences de premier ordre pour une solution $2.0 \mathrm{~mol} \mathrm{~kg}^{-1}$ en $\mathrm{NiCl}_{2}$ dans le $\mathrm{D}_{2} \mathrm{O} .[9,17]$

Nous avons vu le cas d'un cation et d'un anion, où l'interaction de la charge avec le dipôle de l'eau produit des orientations bien définies de l'eau dans la première sphère de coordination. Tournonsnous maintenant vers un soluté non chargé et hydrophobe, l'argon [18, 19]. La figure 7 montre la différence de premier ordre $G_{\mathrm{Ar}}(r)$ obtenue par la substitution de l'argon naturel par le ${ }^{36} \mathrm{Ar}$ dans $1 \mathrm{le} \mathrm{D}_{2} \mathrm{O}$ à $25^{\circ} \mathrm{C}$ (sous une pression d'argon de 230 à 240 bars). Nous ne voyons plus de pics séparés soluté-O et 
soluté-H, ce qui indique que les molécules n'ont pas d'orientation bien définie par rapport à l'argon. II est probable que l'orientation des molécules d'eau est déterminée plus par leur besoin de former des ponts hydrogènes avec les molécules d'eau voisines que par l'interaction avec l'argon. Le fait que l'on voie malgré tout une sphère de coordination assez bien définie atteste de l'importance des interactions répulsives en plus des interactions attractives pour déterminer la structure dans les phases condensées. Il est intéressant de comparer $\mathrm{K}^{+}, \mathrm{Cl}^{-}$et $\mathrm{Ar}$ qui sont isoélectroniques. Les fonctions $G_{\mathrm{Ar}}(r)$ et $G_{\mathrm{K}}(r)$ obtenues en solution dans le $\mathrm{D}_{2} \mathrm{O}$ sont en fait très semblables dans la région de la première sphère d'hydratation (figure $7 b$ ), ce qui indique que la charge de $\mathrm{K}^{+} \mathrm{n}$ 'a que peu d'effet sur les orientations des molécules d'eau dans la première sphère de coordination. Par contre nous avons vu que la première sphère d'hydratation du $\mathrm{Cl}^{-}$est relativement structurée, conséquence de la force relativement élevée de l'interaction $\mathrm{Cl}-\mathrm{H}$, qui est essentiellement une interaction de pont d'hydrogène.

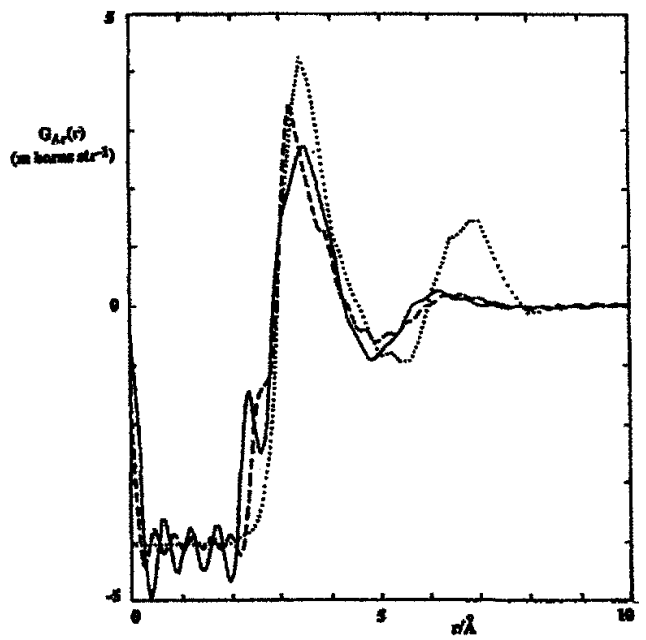

a)

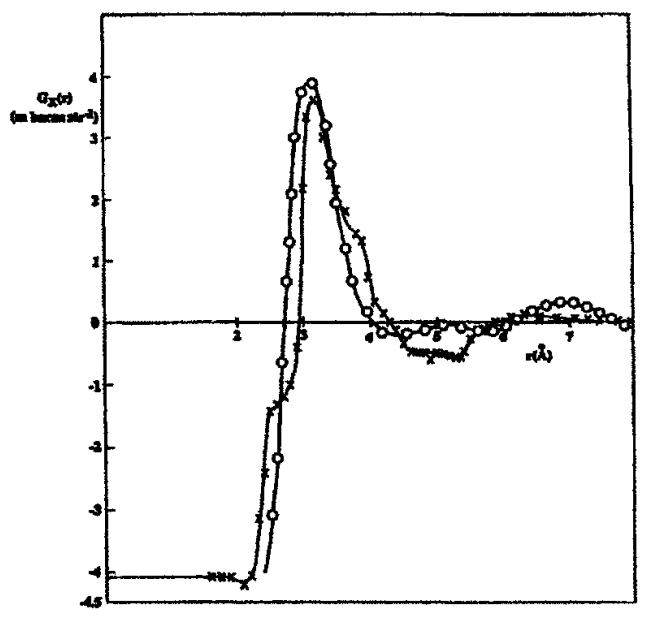

b)

Figure 7 : (a) La fonction de différence de premier ordre, $G_{\mathrm{Ar}}(r)$, obtenue pour une solution dans le $\mathrm{D}_{2} \mathrm{O}$ sous pression d'argon [18]. La courbe pleine et la courbe en traitillés représentent respectivement les résultats sous pression d'argon de 230 et 240 bar. La courbe en pointillés est calculée d'après des travaux théoriques [20]. (b) Comparaison entre $G_{\mathrm{Ar}}(r)$ (croix) et la fonction de différence de premier ordre $G_{\mathrm{K}}(r)$ (cercles) obtenue pour une solution $4.0 \mathrm{~mol} \mathrm{~kg}^{-1}$ en KCl dans le $\mathrm{D}_{2} \mathrm{O} .[18,21]$

Il y a un grand intérêt à comprendre le rôle des ions hydratés dans la dénaturation des protéines. Dans ce but, Wilson et al [22] ont utilisé la substitution isotopique du chlore pour mesurer la fonction $G_{\mathrm{Cl}}(r)$ d'une solution du hydrochlorure de guanadine $(\mathrm{GdmCl})$. La figure 8 montre l'effet de l'addition du polypeptide triglycine à la solution. Le premier pic est mieux défini en présence du polypeptide, 
sans que le nombre d'hydratation soit significativement modifié. Cet effet de renforcement de la structure de la première sphère d'hydratation du chlorure ressemble à celui observé dans des solutions aqueuses du chlorure de lithium sous forme de verre [23]. Ceci laisse supposer que l'eau proche de l'interface avec le polypeptide adopte une structure qui ressemble à celle d'un verre et que cette eau interfaciale est moins mobile que l'eau normale.

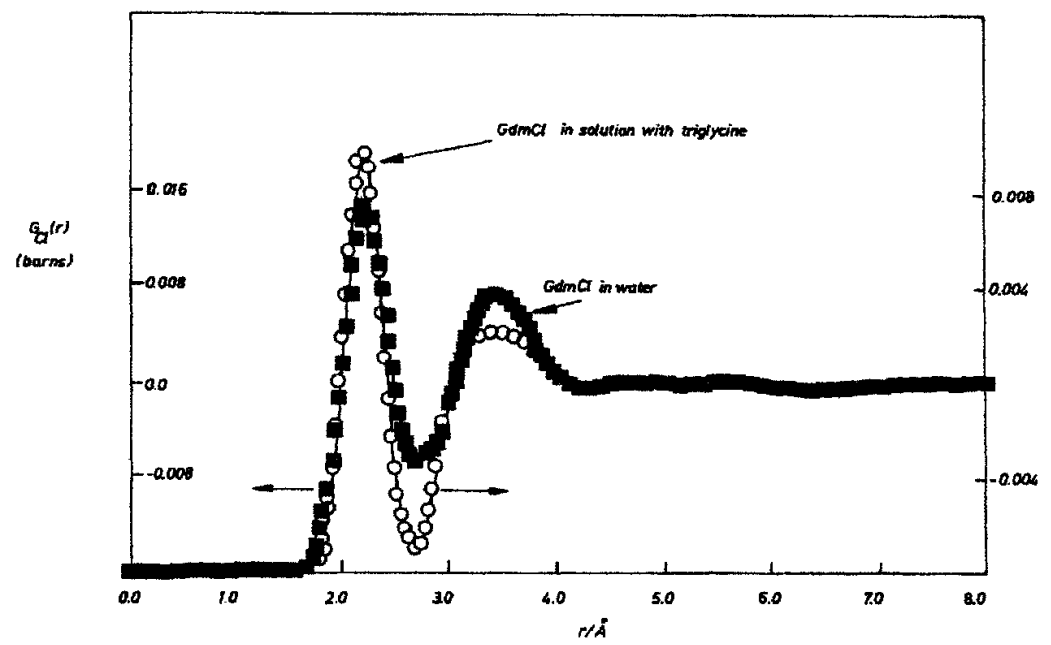

Figure 8: La fonction de différence de premier ordre, $G_{\mathrm{Cl}}(r)$, obtenue pour une solution $4.0 \mathrm{~mol} \mathrm{~kg}{ }^{-1}$ en GdmCl (carrés pleins) et pour une solution $2.0 \mathrm{~mol} \mathrm{~kg}^{-1}$ en $\mathrm{GdmCl}$ et $0.66 \mathrm{~mol} \mathrm{~kg}^{-1}$ en triglycine (cercles ouverts), les denx dans le $\mathrm{D}_{2} \mathrm{O}$ [22].

Un système où nous pourrions également attendre un effet de renforcement de la structure de l'eau proche d'une interface est l'argile montmorillonite. Ce minérale est un composé important des terreaux et des roches et est présent en état collö̈dal dans les eaux naturelles. Il consiste en entassements réguliers de couches ou lamelles cristallines aluminosilicates de charge négative. Entre ces lamelles cristallines se trouvent des cations échangeables (qui contrebalancent la charge négative des lamelles) et de l'eau interlamellaire, qui a une structure relativement liquide (figure 9a). Pitteloud $e t$ al [24] ont étudié l'hydrate à deux couches du Ni-montmorillonite; c'est à dire un montmorillonite où le cation interlamellaire est le $\mathrm{Ni}^{2+}$ et l'espacement entre les lamelles est suffisamment grand pour permettre deux couches d'eau interlamellaire. Ce système peut être décrit comme une solution aqueuse bidimensionelle du $\mathrm{Ni}^{2+}$, d'épaisseur d'environ $7 \AA$, où l'anion est la surface des lamelles de l'argile. Ces auteurs ont combiné la substitution isotopique du nickel et de l'hydrogène de l'eau $\left(\mathrm{H}_{2} \mathrm{O}\right.$ où $\mathrm{D}_{2} \mathrm{O}$ comme eau interlamellaire) pour obtenir la fonction de distribution de paires partielle, $g_{\mathrm{NiH}}(r)$, qui décrit l'hydratation $\mathrm{du} \mathrm{Ni}^{2+}$ dans la région interlamellaire. La fonction $g_{\mathrm{NiH}}(r)$ obtenue est pratiquement 
identique à la fonction analogue pour une solution aqueuse normale du $\mathrm{Ni}^{2+}$ (figure $9 \mathrm{~b}$ ). De plus, la structure de l'eau interlamellaire déterminée par la diffraction neutronique montre peu de différence par rapport à celle de l'eau pure. Ici donc, la structure de la sphère d'hydratation du cation est déterminée principalement par l'interaction du cation avec l'eau. Les ponts hydrogènes formés par les molécules d'eau avec la surface de l'argile, n'empêchent pas l'eau de former des réseaux de ponts hydrogènes plus ou moins tétraédriques. Se pourrait-il que le chlorure, dont la structure d'hydratation est clairement renforcée par la présence de biomolécules, soit une sonde particulièrement sensible aux modifications de la structure de l'eau autour des interfaces?

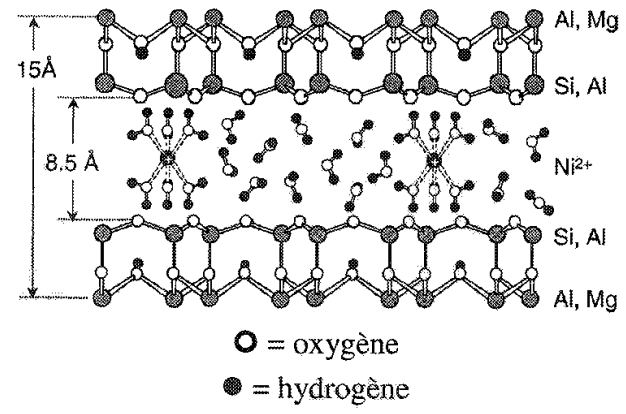

a)

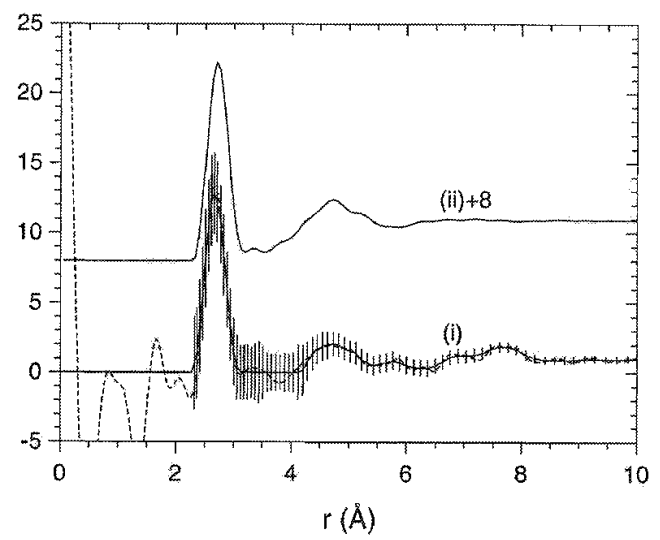

b)

Figure 9 ; (a) Représentation d'une section perpendiculaire aux lamelles aluminosilicates du montmorillonite, montrant les cations échangeables et l'eau dans l'espace interlamellaire. (b) La fonction de distribution de paires partielle, $g_{\mathrm{NiH}}(r)$ obtenu (i) pour le Ni-montmorillonite et (ii) pour une solution aqueuse $2 \mathrm{~mol} \mathrm{~kg}^{-1}$ en $\mathrm{NiCl}_{2}[24]$.

\section{TECHNIQUES COMPLEMENTAIRES}

Considérons d'abord les techniques de rayons $\mathrm{X}$ complémentaires à la diffraction neutronique pour l'étude de solutions aqueuses. La diffraction $X$ simple, tout comme la diffraction neutronique sans substitution isotopique, n'a que peu à offrir à l'étude de solutions aqueuses puisque, à moins que le soluté soit de poids atomique élevé et en concentration élevée, la diffraction est dominée par le solvant. La recherche d'une méthode analogue à la substitution isotopique a d'abord porté sur la substitution isomorphique, qui a été appliqué à des ions en solution aqueuse [25,26]. La méthode suppose que, pour bien des ions, la structure d'hydratation est déterminée principalement par la charge et le rayon ionique de l'ion. Si nous pouvons trouver une paire d'ions de charge identique, et de rayon ionique très proche, nous pouvons les supposer isomorphes. Si de plus la différence de poids atomique est assez grande, nous pouvons soustraire les intensités de diffraction de deux solutions de composition identique 
excepté la substitution des deux solutés isomorphes, et obtenir une fonction de différence premier ordre. Cette fonction sera analogue à celle obtenue par diffraction neutronique avec substitution isotopique, mais approximative et dominée par les corrélations ion-O (la diffusion de rayons $\mathrm{X}$ par l'hydrogène étant extrêmement faible). ${ }^{7}$ Cette méthode a donné des résultats utiles sur les distances moyennes ion-oxygène dans la première sphère de coordination, mais n'est pas considérée comme réellement quantitative au niveau de nombres de coordination.

L'arrivée de sources de synchrotron brillantes a permis l'application de la diffraction anomale de rayons $\mathrm{X}$ (décrite lors d'un cours préalable) à des solutions aqueuses. Dans ce cas, nous n'avons besoin que d'un seul échantillon, et le contraste relatif au soluté $\mathrm{X}$ qui nous intéresse est donné par la variation de son facteur de forme atomique, $f_{X}$, autour d'un seuil d'absorption. La différence des intensités de diffraction à deux énergies différentes près d'un seuil d'absorption de $\mathrm{X}$ nous donne une fonction de différence de premier ordre, $\widetilde{\Delta}_{\mathrm{X}}(Q)$. Cette fonction et sa transformée de Fourier, $\widetilde{G}_{\mathrm{X}}(r)$, sont exactes dans la mesure où nous pouvons corriger et normaliser correctement les intensités de diffraction. Suite à la variation des facteurs de forme atomique avec $\mathrm{Q}$, la fonction $\widetilde{G}_{\mathrm{X}}(r)$ n'est pas une simple somme pondérée de $g_{\mathrm{X} \alpha}(r)$, mais une convolution de ceux-ci, dont $g_{\mathrm{Xo}}(r)$ fait la plus grande contribution. La figure 10 montre le résultat d'une expérience de diffraction anomale de rayons $\mathrm{X}$ sur une solution de chlorure d'yttrium autour d'un seuil d'absorption de l'yttrium [27]. La fonction $\widetilde{G}_{\mathrm{Y}}^{*}(r)$ est une forme modifiée de $\tilde{G}_{Y}(r)$ où la plupart des effets de la dépendance en $Q$ des facteurs de forme atomiques ont été éliminés. Nous voyons très clairement le pic $\mathrm{Y}^{3+}-\mathrm{O}$ de la première sphère d'hydratation et une deuxième sphère d'hydratation assez bien définie. Le nombre d'hydratation de 8 obtenu avec ces

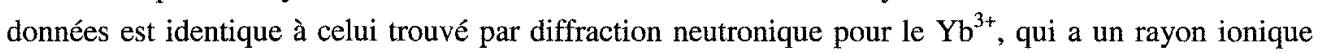
très semblable [28]. La diffraction anomale offre donc la possibilité d'étudier la structure autour des solutés pour lesquels il n'y a pas d'isotopes à longueurs de diffusion neutronique suffisamment distinctes pour permettre des expériences de différence en diffraction neutronique. De plus, le fait que $\widetilde{G}_{\mathrm{X}}(r)$ est dominé par les corrélations X-O peut être utile dans des cas où les corrélations X-O et X-H ne sont pas facilement séparables par des mesures de diffraction neutronique. Il faut ajouter que des mesures de diffraction anomale sont très exigeantes au niveau expérimental et sont limitées pour le moment à des solutions relativement concentrées. ${ }^{8}$

\footnotetext{
${ }^{7}$ La substitution isomorphique est également applicable à la diffraction neutronique. Dans ce cas, les erreurs dues à l'approximation isomorphique imparfaite sont en géneral plus marquées à cause de la pondération moins forte des contributions soluté-solvant à $F(Q)$.

${ }^{8}$ Du point de vue chimique, il est préferable de travailler en solution diluée pour éviter des problèmes de formation d'ions complexes où d'hydroxydes et pour mieux définir le $\mathrm{pH}$ de la solution. De plus, bien de solutés intéressants ne sont que faiblement solubles.
} 


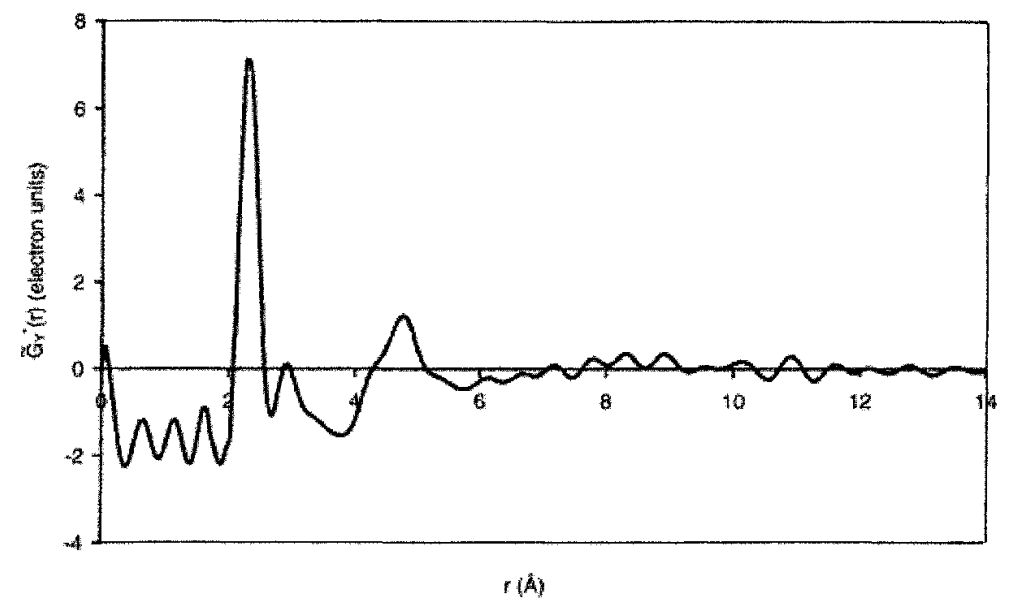

Figure 10 : Fonction de différence de premier ordre modifiée, $\widetilde{G}_{\mathrm{Y}}^{*}(r)$, obtenue par la diffraction anomale de rayons $\mathrm{X}$ pour une solution $3.5 \mathrm{~mol} \mathrm{~kg}^{-1}$ en $\mathrm{YCl}_{3}$ [27]

Une autre méthode qui utilise le rayonnement de synchrotron et qui a été largement appliquée à des solutions aqueuses est la spectroscopie EXAFS (structure fine dans l'absorption de rayons $\mathrm{X}$ étendue) [29]. Dans une expérience d'EXAFS nous mesurons le spectre absorption $X$ en fonction d'énergie à partir d'un seuil d'absorption de l'élément qui nous intéresse. La structure fíne de cette absorption en fonction de l'énergie peut être convertie en une fonction de $Q$ et puis une fonction de distribution radiale autour de l'élément visé. Dans le cas de solutions aqueuses nous pouvons donc obtenir la distance entre le soluté qui nous intéresse et la première sphère de coordination et, avec une précision qui dépende de la qualité du modèle utilisé, des nombres de coordination. L'EXAFS est considéré par beaucoup de chercheurs comme étant moins exacte que la diffraction, puisque l'analyse des données doit toujours passer par l'ajustement de modèles au signal d'EXAFS. Par contre, cette méthode offre le grand avantage d'être relativement simple, sensible et rapide, ce qui la rend particulièrement adaptée à des études de solutions peu concentrées ainsi qu'à des mesures multiples en fonction de variables comme la température ou la pression. Puisqu'elle n'est pas nécessaire de connaître très précisément la composition chimique de l'échantillon, la méthode est aussi bien adapté à des études de systèmes complexes et difficiles à préparer de façon reproductible, par exemple dans des applications biologiques ou en science de matériaux.

Finalement, d'autres méthodes spectroscopiques donnent des informations structurales sur des solutés et leurs sphères d'hydratation. Par exemple la résonance magnétique nucléaire (la RMN) [30][31] et la spectroscopie de luminescence [32,33] peuvent, sous de bonnes conditions, donner des 
informations sur des nombres d'hydratation en solution. La RMN a également été largement appliqué à l'étude de la structure en solution et de l'hydratation des protéines et d'autres biomolécules $[34,35]$.

\section{CONCLUSIONS}

En conclusion, la diffraction neutronique, grâce à la possibilité d'utiliser des substitutions isotopiques a contribué, et continuera à contribuer, largement à nos connaissances de l'eau et des solutions aqueuses. La disponibilité d'appareillages de plus en plus performants permettra sûrement l'application plus large de méthodes de substitution isotopique multiple pour mieux. préciser nos connaissances de systèmes aqueux et d'aborder de nouvelles questions dans ce domaine. D'autres méthodes, dont les techniques de rayonnement de synchrotron qui sont proches de la diffraction neutronique, contribueront à ces avances en nous permettant d'étudier des systèmes qui ne sont pas abordables par diffraction neutronique et en nous donnant des informations complémentaires sur des systèmes qui le sont.

\section{Références}

1. V.F. Sears, Neutron News 3, 26 (1992); les mêmes informations sont disponibles à http://www.ncnr.nist.gov/resources/n-lengths.

2. K.E. Gubbins, C.G. Gray, P.A. Egelstaff, M.S. Ananth, Mol. Phys. 25, 1353 (1973).

3. J.C. Dore, J. de Physique C7, 49 (1984).

4. A.K. Soper, M.G. Phillips, Chem. Phys. 107, 47 (1986).

5. A.K. Soper, Chem. Phys. 107, 61 (1986).

6. Voir http://www.isis.rl.ac.uk/Disordered/SANDALS.htm pour plus d'informations sur le diffractomètre SANDALS.

7. G. Placzek, Phys. Rev. 86, 377 (1952).

8. H.E. Fischer, P.S. Salmon, A.C. Barnes, 'La diffraction des neutrons et des rayons X pour l'étude des liquides et des verres', Proceedings de l'école thématique 'Neutrons et Matériaux', Trégastel, 14-16 mai 2001 ; à paraître dans le Journal de Physique.

9. D.H. Powell, G.W. Neilson, J.E. Enderby, J. Phys.: Condens. Matter 1, 8721 (1989).

10. A.K. Soper and A. Luzar, J. Chem. Phys. 97, 1320 (1992).

11. H.E. Fischer, Habilitation à Diriger les Recherches, Université Joseph Fourier Grenoble I (1997).

12. R.L. McGreevy, L. Pusztai Molec. Simul. 1, 359 (1988).

13. A.K. Soper, Chem. Phys. 258, 121 (2000).

14. A.K. Soper, Physica B 276, 12 (2000).

15. A.K. Soper, G.W. Neilson, J.E. Enderby, R.A. Howe, J. Phys. C. 10, 1793 (1977).

16. D.H. Powell, A.C. Barnes, J.E. Enderby, G.W. Neilson, P.S. Salmon, Faraday Discuss. Chem. Soc.

85, 137 (1988).

17. D.H. Powell, G.W. Neilson, J.E. Enderby, J. Phys.: Condens. Matter 5, 5723 (1993). 
18. R.D. Broadbent, G.W. Neilson, J. Chem. Phys. 100, 7543 (1994)

19. D.M. Sullivan, G.W. Neilson, J. Chem. Phys. 115, 339 (2001).

20. C. Pangali, M. Rao, B.J. Berne, J. Chem. Phys. 71, 2982 (1979).

21. G.W. Neilson, N.T. Skipper, Chem. Phys. Lett. 114, 35 (1985).

22. J.E. Wilson, S. Ansell, J.E. Enderby, G.W. Neilson, Chem. Phys. Lett. 278, 21 (1997).

23. S. Ansell, J. Dupuy-Philon, J.-F. Jal, G.W. Neilson, J. Phys.: Condens. Matter 9, 8835 (1997).

24. C. Pitteloud, D.H. Powell, H.E. Fischer, Phys. Chem. Chem. Phys. 3, 5567 (2001).

25. N.T. Skipper, G.W. Neilson, S. Cummings, J. Phys.: Condens. Matter 1, 3489 (1989).

26. N T. Skipper, G.W. Neilson, J. Phys.: Condens. Matter 1, 4141 (1989).

27. S. Ramos, G.W. Neilson, A.C. Barnes, A. Mazuelas, J. Phys. Chem. B 105, 2694 (2001).

28. C. Cossy, L. Helm, D.H. Powell, A.E. Merbach, New J. Chem. 19, 27 (1985).

29. A. Filipponi, J. Phys. Condens. Matter 13, R23 (2001).

30. M.C. Alpoim, A.M. Urbano, C.F.G.C. Geraldes, J.A. Peters, J. Chem. Soc. Dalton Trans. 3, 463 (1992).

31. E.J. Sutter, D.M. Updegrove, J. F. Harmon, Chem. Phys. Lett. 36, 49 (1975).

32. W.deW. Horrocks Jr., D.N. Sudnick, Acc. Chem. Res. 14, 384 (1981).

33. G.R. Choppin, D.R. Peterman, Coord. Chem. Rev. 174, 283 (1998).

34. M. Pfuhl, P.C. Driscoll, Phil. Trans. Roy. Soc. London 358, 513, (2000).

35. P.S. Belton, Cellular \& Molecular Life Sci. 57, 993 (2000). 